\title{
Thermal Analysis of Polyurethane Dispersions Based on Different Polyols
}

\author{
Suzana M. Cakić, Ivan S. Ristić and Olivera Z. Ristić
}

Additional information is available at the end of the chapter

http://dx.doi.org/10.5772/35800

\section{Introduction}

\subsection{Water-based polyurethane dispersions}

Water-based polyurethane dispersions (PUD) are a rapidly growing segment of polyurethane (PU) coatings industry due to environmental legislations such as the clean air act and also due to technological advances that made them an effective substitute for the solvent-based analogs. Water-based or waterborne PUD have gained increasing importance in a range of applications, due in large part to properties such as adhesion to a range of substrates, resistance to chemicals, solvents and water, abrasion resistance and flexibility. Water-based PUD show very good mechanical and chemical properties and match the regulatory pressures for low volatile organic compound (VOC) containing raw paints. The continuous reduction in costs and the control of VOC emissions are increasing the use of water-based resins, motivating the development of PU dispersed in water. PU obtained from water-based PUD have superior properties when compared with similar materials obtained from organic media. Water-based PUD are used in many application areas to coat a wide range of substrates - for example footwear adhesives, wood lacquers for flooring and furniture, leather finishings, plastic coatings, printing inks and automotive base coats (Rothause et al., 1987; Kim et al., 1994; Ramesh et al., 1994).

Regarding the chemical nature of PU, the water based PU are applied with higher solids content, compared to the solvent based PU, because their viscosity does not depend on the molecular weight of PU, as is the case for solvent based PU (Gunduz \& Kisakurek, 2004). Thus waterborne PUD can be prepared at high solid contents with a molecular weight enough to form films with excellent performance resulting solely upon "physical drying". This means that the film formation occurs by simple evaporation of water even at room temperature. 
Waterborne PUD are fully-reacted PU systems produced as small discrete particles. 0.1 to 3.0 micron, dispersed in water to provide a product that is both chemically and colloidally stable, which only contains minor amounts of solvents and does not emit VOC. Polymeric structure of waterborne PUD is formed by usually reacting an excess of aliphatic isocyanates (mainly IPDI or HDI based), with a polyol or a mixture of polyols to form a prepolymer containing the so called soft segment. The polyols are generally polyesters, polyethers, or polycarbonates. The hard segment is generally formed by chain extending the prepolymer with short chain diamines and from the short chains containing ions. Due to incompatibility between the two segments of the polymer chain, the hard segment separates and aggregates into domains that act as reinforcing fillers to the soft segment. The degree of phase separation as well as the the concentration of the hard segments are contributing factors to the good properties of PUD. PU backbone with a minority of the repeat units contains pendant acid or tertiary nitrogen groups, which are completely neutralized or quarternized, respectively, to form salts. Such ionomeric groups are absolutely necessary for the formation of dispersions, because they act as internal surfactants, and are not incorporated in the chain of the solvent-based PU. Ionic centers in the hard segment generally favor segregation and cohesion within the hard segment domains due to their strong electrostatic forces and thermodynamic incompatibility with the polymer matrix. Water-based PUD can be divided into two classes. The first group consists of polymers stabilized by external emulsifiers, and second one achieves stabilization by including hydrophilic centers in the polymer. Such hydrophilic centers may be one of the three types: non-ionic, cationic groups and anionic groups. These hydrophilic groups fulfill the function as internal emulsifiers and make possible to produce stable water/based emulsions. Water-based PUD can be classified into anionic, cationic and nonionic systems (Rothause \& Nechtkamp, 1987; Kim et al., 1996).

Several processes have been developed for the synthesis of PUD. All of these have in common the first step, in which a medium molecular weight polymer (the prepolymer) is formed by the reaction of suitable diols or polyols (usually macrodiols such as polyether or polyester) with a molar excess of diisocyanates or polyisocyanates. In this reaction mixture, an internal emulsifier is added to allow the dispersion of the polymer in water; this emulsifier is usually a diol with an ionic group [carboxylate, sulfonate, or quaternary ammonium salt) or a nonionic group poly(ethylene oxide)]. The internal emulsifier becomes part of the main chain of the polymer. The critical step in which the various synthetic pathways differ is the dispersion of the prepolymer in water and the molecular weight build-up. The most important dispersions are emulsifier-free ionomer dispersions. The resulting dispersions are mainly anionic or non-ionic, that have the potential for wide variations in composition and property level. They can be obtained by different processes, however, the earliest process to prepare the aqueous PUD is known as acetone process and this process has remained technically important so far (Hepburn, 1992; Oertel, 1985). Within the last three decades several new processes have been developed such as prepolymer mixing process, hot melt process and ketamine/ketazine process.

The facts that aqueous/water PUD have become increasingly important for industrial and academic research in recent years is due to the following reasons: 
1. the environmental law requires for the development of ecological-friendly products for which the emissions of volatile organic compounds (VOC) have been reduced to a minimum,

2. the economic reasons (substitution of expensive organic solvents in conventional PU with water),

3. the water PU surpasses performance of conventional isocyanate- and/or solventcontaining PU,

4. continuous increase in solvent prices, low raw material costs and easy to clean up the reactor system made waterborne PU system more popular in the industry.

\section{Ingredients for water-based PUD}

The basic components used to build up PUD include long-chain polyether, polyester or polycarbonate polyol, diisocyanate, aromatic or (cyclo)aliphatic, low molecular weight glycol and /or diamine, bis-hydroxycarboxylic acid and a neutralization base. In general, an excess of diisocyanate is treated with a long-chain linear polyol, bis-hydroxycarboxylic acid and other low-molecular-weight glycol to form an isocyanate-terminated prepolymer with a segmented structure. In this polymer, the long-chain polyol units form soft segments, and the urethane units-built up from diisocyanate, glycol and bis-hydroxycarboxylic acid form hard segments. The pendant carboxylic acid groups are neutralized with base to form internal salt group containing prepolymers that can be easily dispersed in water. The microphase separation between the incompatible soft- and hard-segment sequences contributes to the unique properties of PUD. The PU chains with NCO terminating groups can be extended with glycol forming urethane groups. Chain extenders are low molecular weight, hydroxyl and amine terminated. Aliphatic isocyanates: hexamethylene diisocyanate (HDI), isophorone diisocyanate (IPDI) and (4,4'-diisocyanatodicyclohexylmethane ( $\left.\mathrm{H}_{12} \mathrm{MDI}\right)$, improve thermal and hydrolytic stability, resistance to UV degradation and they do not yellow (Bechara, 1998).

Aliphatic diisocyanates are less reactive than aromatic ones and they must be used with certain catalysts. Aromatic isocyanates: methylene diphenyl diisocyanate (MDI), toluene diisocyanate (TDI) and 1,5-naphthalenediisocyanate (NDI) on the other hand, provide for toughness but yellow upon exposure to UV light. Although early water dispersed PU resins heavily utilized TDI, there is a high tendency to shift to aliphatic diisocyanates or to the aromatic diisocyanates with NCO groups not directly attached to an aromatic nucleus (Gunduz \& Kisakurek, 2004).

The two key classes of polyols are polyethers and polyesters. Polyester polyols have been largely used in PUD paints as they exhibit outstanding resistance to light and aging. There are four main classes of polyester polyols: linear or lightly branched aliphatic polyester polyols (mainly adipates) with terminal hydroxyl groups, low molecular weight aromatic polyesters for rigid foam applications, polycaprolactones, polycarbonate polyols. Polyether polyols are susceptible to light and oxygen when hot, however, they improve water dispersion, and impart chain flexibility. These are made by the addition of alkylene oxides, 
usually propylene oxide, onto alcohols or amines which are usually called starters or 'initiators'. Polyether based on propylene oxide contains predominantly secondary hydroxyl end-groups. Secondary hydroxyl end-groups are several times less reactive with isocyanates than primary hydroxyl groups and for some applications, polyether based only on propylene oxide may have inconveniently low reactivity. The primary hydroxyl content may be increased by a separate reaction of the polyoxypropylene polyols with ethylene oxide to form a block copolymer with an oxyethylene tip.

In the choice of polyol for PU application, selected polyols must be competitive with other polyols and also enable the final PU product to be cost competitive with other materials in the end application.

In a typical anionic PUD process, anionic groups (carboxylic and sulfonic) are introduced along the length of the polymer chain by using hydrophilic monomers or internal emulsifiers. As the hydrophilic monomer, dimethylol propionic acid (DMPA) is the most widely used acid, which has two hydroxyl groups, therefore, it can be one of the main constituents of the PU backbone. DMPA improves the hydrophilic property by serving as the potential ionic center with N-methyl pyrrolidone as the co-solvent. Tartaric acid (TA) can also be used, but it usually results in branching. However, TA improves the mechanical properties of PU paint. Study has shown that the particle size of dispersion depends on the content of DMPA. Therefore, increased amount of DMPA leads to more ionic centres in the PUD backbone and thereby increasing hydrophilicity of the polymer and hence reductions in particle size (Dieterich, 1981; Jacobs \& Yu, 1993; Rosthauser \& Nachkamp, 1986).

The chain extension step also has a high influence on the properties of the resin produced, not only due to the structure and concentration of the extender but also due to process variables that influence the particle size distribution. Chain extenders are difunctional glycols, diamines or hydroxyl amines. If a diol of low molecular weight reacts with the NCO terminated PU chains in the chain extension reaction step, urethane linkages will be formed but if a diamine is used as chain extender, the NCO terminated PU chains will form urea linkages. The higher density of hydrogen bonds of polyurea hard segments is responsible for the improved mechanical properties of polyurea and PU/urea products. Typical chainextending agents are as follows: water, diethylene glycol, hydroquinone dihydroxyethyl ether, bisphenol A, bis(hydroxyethylether), ethanolamine, hydrazine, ethylene diamine. Aliphatic diamines such as hydrazine or ethylene diamine are used as chain extenders in processes directed to preparing waterborne PUD. In the chain extension step, it is most important to control the extremely fast reaction between $\mathrm{NCO}$ groups and $\mathrm{NH}_{2}$ groups accompanied by the viscosity rise. Molecular weight of PUD increases by the formation of urea linkages through the chain extension step and it is the most important step to determine the molecular weight of PUD (Kim, 1996; Delpech \& Coutinho, 2003).

In the synthesis of PUD, to neutralize the carboxyl and/or sulfo groups, are used agents that contain one or more bases and to form internal salt groups containing prepolymers that can be easily dispersed in water. During the neutralization, carboxyl and/or sulfo groups serve for anionic modification or stabilization of the PUD. Tertiary amines and in particular 
triethylamine are preferably used. The structure of waterborne anionic PUD is illustrated in Scheme 1 .

Most commonly used catalysts in PU chemistry are tertiary amine catalysts and metal catalysts, especially tin catalysts. Tertiary amines are catalysts for the isocyanate-hydroxyl and the isocyanate-water reactions. Organotins are the most widely used, however organomercury and organolead catalysts are also used but have unfavourable hazardous properties.

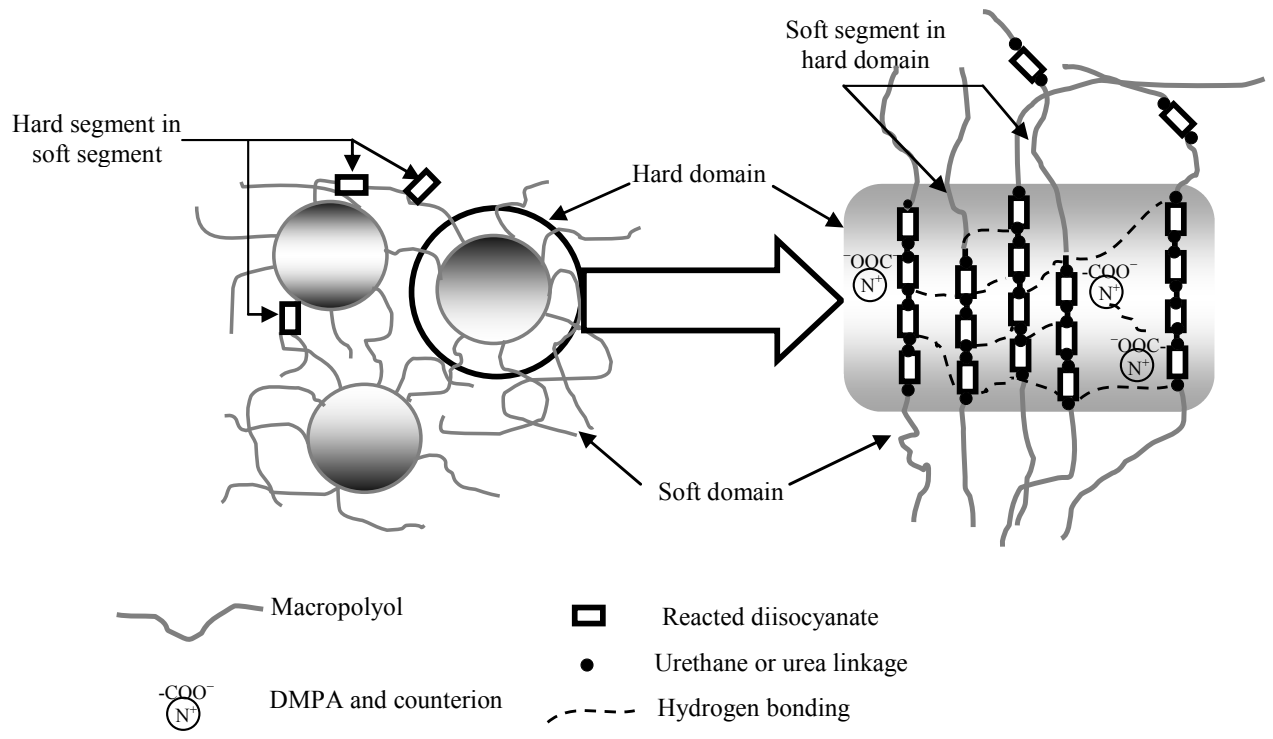

Scheme 1. Structure of waterborne anionic PUD (Tawa \& Ito, 2006)

\subsection{Various methods for preparing water-based PUD}

The most important process is the prepolymer mixing process that has the advantage of avoiding the use of a large amount of organic solvent. In this process hydrophilically (carboxylate molecule) modified prepolymer is directly mixed with water. If the mixture viscosity is too high, a small amount of a solvent such as $\mathrm{N}$-methyl pyrrolidone can be added before the dispersion step. Chain extension is accomplished by the addition of di- or polyamines to the water-based prepolymer dispersion.

The acetone process can be considered the link between the solvent synthesis and the prepolymer mixing process. In effect, the prepolymer is synthesized in a hydrophilic organic solvent, for example acetone solution and afterwards it is subsequently mixed with water.

The hot melt process explains the process of obtaining a PUD by the reaction of NCOterminated ionic modified prepolymer with, for example ammonia or urea resulting in a prepolymer with terminal urea or biruet groups, respectively. The terminal urea or biruet prepolymer is methylolated with formaldehyde and mixed with water, forming dispersion 
spontaneously. By polycondensation (lowering the $\mathrm{pH}$, increasing the temperature), chainextension or cross-linking was obtained.

Ketamine and ketazine process explains the process of obtaining a PUD by reaction of NCO-prepolymers containing ionic groups mixing with a blocked amine (ketamine) or hydrazine (ketazine) without premature chain extension. These mixtures can be emulsified with water even in absence of co-solvents. The reaction with water liberates the diamine or hydrazine, which then reacts with the prepolymer.

Non-ionic dispersions are obtained similar to ionomer dispersions if the ionic centre is replaced by lateral or terminal hydrophilic ether chain. The temperature of dispersing process has to be kept below $60{ }^{\circ} \mathrm{C}$. Non-ionic dispersions are stable towards freezing, $\mathrm{pH}$ changes and addition of electrolytes.

\section{Thermal analysis of PU}

Thermal analysis techniques have been used for many years in many scientific and industrial laboratories for studying the thermal decomposition of polymeric materials. Among them thermogravimetry (TG) is one of the most common since the mass of a sample is easy to measure accurately and valuable information regarding the nature of the process can be extracted from a mass loss against time or temperature plot. The thermal decomposition of PU (their degradation attributed to absorbed thermal energy) is important phenomenon from both fundamental and industrial applications (Pielichowski et al., 2004). The understanding of degradation processes allows determination of optimum conditions for designing PU in order to obtain high-performance polymer materials. Fundamental research has established that the thermal decomposition of PU is a complex heterogeneous process and consists of several partial decomposition reactions (Scaiano, 1989). The study of the decomposition of PU is particularly difficult since they degrade with the formation of various gaseous products and a number of decomposition steps are typically observed in thermogravimetric analysis (TGA) experiments. Some authors claim that the study of the thermo-degradation behavior of $\mathrm{PU}$ at high temperatures provides a fingerprint of the material that has to do not only with the characteristics of the original material, but also with its processing and the final quality of the end use products (Prime et al., 1988). The thermal stability of a material is defined by the specific temperature or temperature-time limit within which the material can be used without excessive loss of properties (Chattopadhyay \& Webster, 2009). With respect to commercial applications, the investigation of thermal decomposition processes has two important aspects. The first concerns the stabilization of a polymer to obtain novel materials with a desired level of thermal stability that will be able to fulfill the demands of contemporary materials engineering. The second is to understand material behavior at higher temperature as well as to obtain characteristic thermal decomposition data.

A waterborne PUD can be defined as a binary colloidal system in which PU particles are dispersed in a continuous aqueous medium. PUD are usually prepared as low molecular weight NCO-terminated prepolymers for ease of dispersion. Then, diamines are generally 
used to increase the molecular weight by reaction with the terminated NCO end groups (chain extension). The presence of ionic species in PUD has a considerable effect on the physical properties. PUD are now one of the most rapid developing and active branches of PU chemistry.

PU are synthesized by the prepolymer reaction of a diisocyanate and a polyol (mainly polyethers and polyesters). If a diol of low molecular weight reacts with -NCO-terminated prepolymers in the chain extension reaction step, urethane linkages will also be formed but if a diamine is used as chain extender, the reaction between the $-\mathrm{NH}_{2}$ groups and the $-\mathrm{NCO}$ terminated prepolymers will form urea linkages. In this case, poly(urethane-urea)s, which are the most important class of polyureas, are produced. These copolymers show reduced plasticity in comparison to homopolyurethanes. The resulting PU or poly(urethane-urea) chains consist of alternating short sequences forming soft (flexible) and hard (rigid) segments. The soft segments, originated from the polyol, impart elastomeric characteristics to the polymer. The hard segments are mainly produced by reacting the isocyanate and the chain extender. They are polar and impart mechanical properties to PU. The hard segments contain the highly polar urethane linkages. Due primarily to interurethane and urea hydrogen bonding, the two segment types tend to phase-separate in the bulk, forming microdomains. The hard segments act as physical crosslinks and, as a consequence, the physical, mechanical and adhesive properties depend strongly on the degree of phase separation between hard and soft segments and interconnectivity of the hard domains. The urethane linkages in PU can serve as H-bond acceptor and donor. In polyether-based PU, the urethane $-\mathrm{NH}$ can bond to either the polyether $-\mathrm{O}-$ linkage or the urethane $-\mathrm{C}=\mathrm{O}$ groups. In the case of poly(urethane-urea) formation, there is an additional -NH from urea linkage participating in the interactions (Delpech \& Coutinho, 2000). The degradation of thermoplastic PU has been extensively studied, and a number of reviews are available (Lu et al., 2002; Fambri et al., 2000). Thermal degradation of ester- and ether-based thermoplastic PU is performed under vacuum, air and nitrogen, allowing investigators to determine the mode of degradation (Dulog \& Storck, 1996).

Polyester-based thermoplastic PU exibit rapid degradation in air and nitrogen, indicating that a nonoxidative mechanism is involved. In contrast, the significantly improved thermal stability of ether-based PU under vacuum and nitrogen indicates that the oxidative process plays a major role in the decomposition of ether-based thermoplastic PU. In general, the ester-based PU normally exibit better thermal and oxidative stabilities than the ether-based ones. The mechanism of thermal degradation of PU is very complex due to the variety of products formed.

It is proposed that the thermal degradation of thermoplastic PU is primarily a depolycondensation process, which starts at about $200{ }^{\circ} \mathrm{C}$ (Cakić et al., 2006 a). The first stage of decomposition is because of degradation of hard segments and starts at about 200 ${ }^{\circ} \mathrm{C}$ and at $\sim 360-380{ }^{\circ} \mathrm{C}$, while the second step of degradation is because of degradation of soft segments and ends above $480^{\circ} \mathrm{C}$. Waterborne PU should exhibit some different features in thermal degradation due to their unique chain structure, for example, salt-forming groups. Therefore, it is necessary to analyses their thermal degradation behavior to 
understand the structure-property relationship. The properties of PUD are mainly determined by the interactions between the hard and soft segments, and by the interactions between the ionic groups. The ionic group content, solids content, segmented structure, molecular weight of the macroglycol, the type of chain extender and the hard/soft segments ratio, determine the properties of PUD.

In the following sections, we will review typical results to demonstrate the utility of TGA in deducing the structural and bonding information about waterborne PUD based on different polyols. The thermal stability of PU and poly(urethane -urea)s cast films with anionomer character, obtained from waterborne dispersions and based on isophorone diisocyanate (IPDI), dimethylolpropionic acid (DMPA), poly(propylene glycol) (PPG), polycarbonate diol (PCD) and glycolized products obtained from recycled poly(ethylene terephthalate)(PET) is also compared. Three types of chain extenders were used: ethylene glycol (EG), propylene glycol (PG) and ethylene diamine (EDA). The effect of type of polyols, chain extender, type of catalyst, ionic content, length of soft segment, hard segment content and the presence of urea or urethane linkages on the thermal stability of the waterborne anionic PUD are discussed.

\section{Water-based PUD based on poly(propylene glycol) and selective catalyst}

One of the inherent drawbacks of waterborne PU technology is the formation of carbon dioxide due to the side reactions of isocyanate with water. When an isocyanate reacts with water, the products are a urea linkage (via an amine intermediate) and carbon dioxide. The carbon dioxide formation is problematic in that it causes imperfections in the coating during cure, such as blistering and pin-hole formation. The main aspect in the development of waterborne PU is in the first place to find methods for preventing the undesired secondary reactions with water and achieving the best crosslinking. This reaction is reduced to a minimum by the use of non-tin catalysts. One novel approach to control the water side reaction is the use of catalysts which selectively catalyze the isocyanate-polyol reaction and not the isocyanate-water reaction (Colling et al., 2002; Blank \&Tramontano, 1996).

The relative selectivity $(\mathrm{S})$ obtained from equation $\mathrm{S}=\mathrm{P}$ urethane $/ \mathrm{P}$ urea, was measured as urethane IR peak area (Purethane)/ urea IR peak area (Purea) ratio, by method given by Blank (Blank et al., 1999). After the integration of characteristic absorption max of urethane (1700 $\left.\mathrm{cm}^{-1}, 1540 \mathrm{~cm}^{-1}\right)$ and urea $\left(1640 \mathrm{~cm}^{-1}, 1570 \mathrm{~cm}^{-1}\right)$ was done, the relative selectivity was calculated. The manganese catalyst, a complex of $\mathrm{Mn}(\mathrm{III})$-diacetylacetonatomaleate with various ligands based on acetylacetonate and maleic acid, used in some of the experiments (Stamenković et al., 2003; Cakić et al., 2006), has shown a high selectivity for the isocyanatehydroxyl reaction in comparison to the commercially available zirconium catalyst (Blank et al., 1999). Zirconium catalyst is a proprietary zirconium tetra-dionato complex in the reactive solvent with the metal content of $0.4 \%$.

TG is a suitable method to evaluate the thermal properties of several types of PU elastomers. The thermal stability of PU has been studied extensively because of the great importance of 
this group of materials (Chang et al., 1995). These thermoplastic elastomers generally are not very thermally stable, especially above their softening temperatures (Wang \& Hsieh, 1997), and their mechanism of thermal degradation is very complex due to the variety of products formed. Commonly, it presents a bimodal profile where the first mode is related to the hard segments of PU. Usually, at a low heating rate, the degradation process results in differential weight loss (DTG) curves with several peaks, which is an indication of the complexity of the degradation (Delpech \& Coutinho, 2000).

\subsection{Experimental}

Poly(propylene glycol) (PPG) ), ( $\mathrm{M}_{\mathrm{n}}=1000$, hydroxyl value $111 \mathrm{mg} \mathrm{KOH} / \mathrm{g}$, dried under vaccuum, at $120^{\circ} \mathrm{C}$ ), isophorone diisocyanate (IPDI) and dimethylolpropionic acid (DMPA), were obtained from Aldrich Chemical Co. 1-methyl-2-pyrrolidone (NMP), dimethyl formamide (DMF) and triethyl amine (TEA) were received from Merck (Darmstadt, Germany). Ethylene glycol (EG) and propylene glycol (PG) obtained from Zorka Co.(Šabac Serbia). Dibutyltin dilaurate (DBTDL), was supplied by Bayer AG. Zirconium catalyst (ZrCat) was supplied by King Industries Inc., Norwalk, CT, USA. Manganese catalyst (MnCat) has been used in the reactive diluent with a metal content of $0.4 \%$ (Stamenković et al., 2003; Cakić et al., 2006 b). Water-based PUD from PPG with selective catalyst were prepared using the prepolymer method has been described in detail in our previously work (Cakić et al., 2009).

In the first step, PPG and DMPA were dispersed in DMF to obtain a homogeneous mixture and heated at $70{ }^{\circ} \mathrm{C}$. IPDI and DBTDL were added to the homogenized mixture at $80{ }^{\circ} \mathrm{C}$. An $\mathrm{NCO} / \mathrm{OH}$ equivalent ratio of 3.0 was used. Hard : Soft segment ratio was defined as a ratio between IPDI weight and polyol weight in the starting formulation and is calculated as 1.5. The reaction times were determined by the dibutyl amine back titration method. After obtaining completely NCO terminated prepolymer, the mixture was cooled to $60^{\circ} \mathrm{C}$ and the carboxylic groups were neutralized with TEA (DMPA equiv) dissolved in NMP. In second step the chain extension was carried out with EG or PG. The selective catalysts, ZrCat or MnCat, at concentration of $2 \%$ relative to the resin solids, have been added to the reaction solution. Water was added to the mixture and stirred to obtain dispersion of organic phase in water. The waterborne PUD contains $40 \mathrm{wt} \%$ solids (Cakić et al., 2009). Films were prepared by casting the waterborne dispersions on leveled surfaces and allowing them to dry at room temperature, for 7 days, and then at $60^{\circ} \mathrm{C}$, for $12 \mathrm{~h}$ (Coutinho, 1996, 2003). When chain extenders were EG and PG, the PUD had to be cast in Teflon surfaces due to the high adhesiveness observed on the glass surface, making demoulding impossible. After demoulding, the films were kept into a desiccator to avoid moisture.and polyurethanes were formed.

\subsection{Results and discussion}

Degradation profile of waterborne PUD is influenced by the variation of chain extender presented in Fig.1. It was verified that the thermal stability was influenced by chain 
extender type. In a general way, thermal stability was higher when EG chain extender was used, in comparison to PG, probably because of the higher reactivity of the primary hydroxyl groups. The onset temperatures calculated for the first stage for PU chainextended with EG were about $234^{\circ} \mathrm{C}$ and $140{ }^{\circ} \mathrm{C}$ for PG. Unsymmetrical structure of IPDI enables easier diffusion of EG (Gunduz \& Kisakurek, 2004).

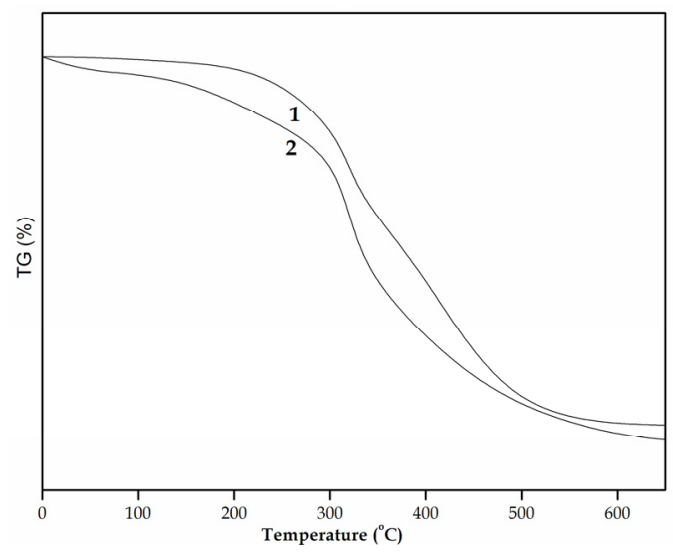

Figure 1. TG curves of PUD without catalyst with EG (1) and PG (2) as chain extender

Fig. 2 shows the degradation profile of PUD with variation of catalyst using catalysts of different selectivity. EG and PG formed urethane linkages by reaction with terminal NCO groups. The initial onsets observed are: $234^{\circ} \mathrm{C}$ when EG was employed, $275^{\circ} \mathrm{C}$ and $311^{\circ} \mathrm{C}$, when the chain extender was EG with ZrCat and EG with MnCat, respectively. The initial onsets observed are: $140{ }^{\circ} \mathrm{C}$ when PG was employed, $290{ }^{\circ} \mathrm{C}$ and $305{ }^{\circ} \mathrm{C}$, when the chain extender was PG with ZrCat and PG with MnCat, respectively. The thermal stability was

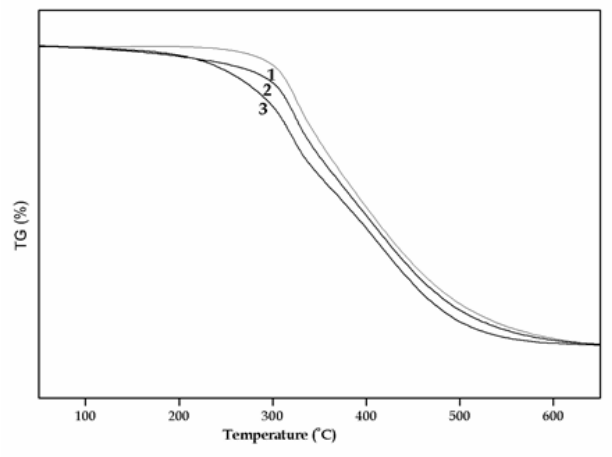

(a)

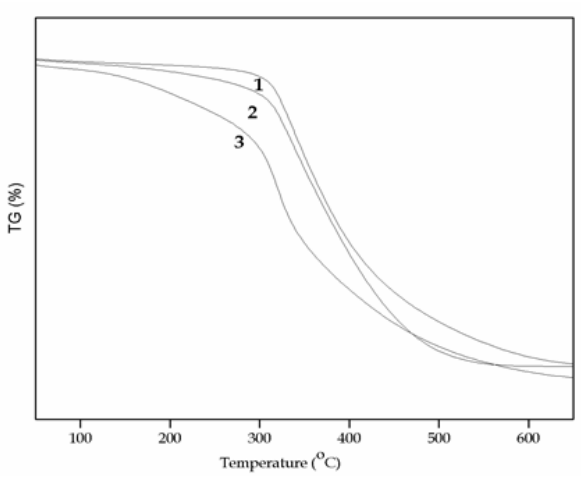

(b)

Figure 2. TG curves of PUD with EG as chain extender (a), PG as chain extender (b), and MnCat (1), ZrCat (2), without catalyst (3) 
higher when MnCat was used, in comparison with the use of ZrCat. This result suggested that all the residual NCO groups in PU particles did not react with the chain extender completely. Because the viscosity of particle is high at low temperature in the chain extension step, it would take long time for chain extenders to diffuse into the particle. Therefore, the efficiency of chain extension increased as total surface area of particles increased (Jang et al., 2002; Cakić et al., 2007 a). In general, the presence of more selective catalyst has also been found to have a stabilizing effect on the resultant PU, as can be observed in the curves obtained for the samples chain-extended with EG and PG, in comparison to the samples obtained without selective catalyst probably due to favoring of isocyanate-polyol reaction and not the isocyanate-water reaction. The PUD was formed with higher hard segment proportions.

The DTG curves show that there are different stages of degradation which are not perceptible in TG curves, showing the close relation and mutual influence between the degradation process of hard and soft segments.

Fig. 3a shows the DTG curves corresponding to the TG degradation profiles presented in Fig. 2a, in which the catalysts were varied (MnCat or ZrCat). The chain extender employed was EG. Two peaks are observed. The first group of peaks, corresponding to the degradation of rigid segments formed by urethane and urea linkages, presents maximum of the peak from 200 to $250{ }^{\circ} \mathrm{C}$. The second group, related to the degradation of PPG soft segment, varying maximum of the peak from 375 to $418{ }^{\circ} \mathrm{C}$. The peaks shifting towards higher temperatures resulting from addition of more selective catalyst confirm the assumption that all isocyanate groups had not reacted with the added chain extender. Selective catalyst isocyanate-polyol reaction causes greater incorporation of chain extender in hard segments, which is reflected on higher thermal stability of hard segments (Jang et al., 2002; Lee et al., 1995; Cakić et al., 2007 b).

Fig. $3 b$ depicts DTG curves related to TG profiles observed in Fig. 2b, in which the catalyst were varied (MnCat or $\mathrm{ZrCat}$ ). The chain extender employed was PG. The first group of peaks presents maximum of the peak appearing in the range from $160{ }^{\circ} \mathrm{C}$ to $195{ }^{\circ} \mathrm{C}$. The

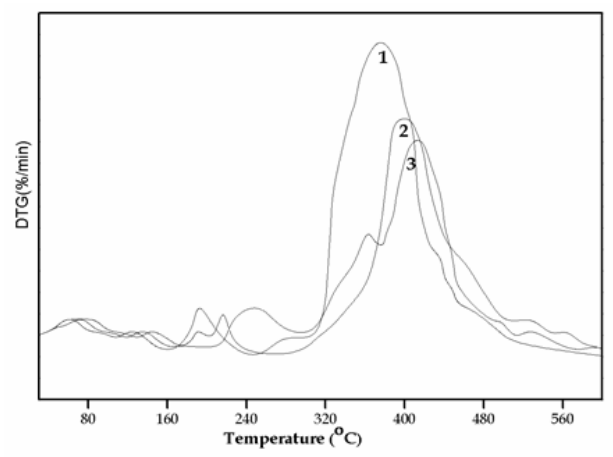

(a)

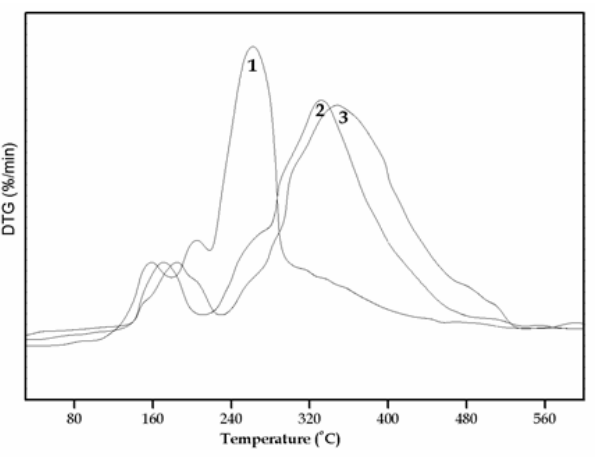

(b)

Figure 3. DTG curves of PUD with EG as chain extender (a), PG as chain extender (b), and without catalyst (1), ZrCat (2), (3) MnCat 
second group can be observed in the range, varying maximum of the peak, from 267 to $347^{\circ} \mathrm{C}$, for PPG soft segments. A marked difference can be observed, promoted by changing the type of chain extender in DTG profiles, especially in the first stage of weight loss, corresponding just to urethane (EG or PG as chain extender) linkage degradation. The soft segment, formed only by PPG degradation step seemed to be also affected. The rigid segment formed from EG retarded the weight loss of PPG chains (peak at $375^{\circ} \mathrm{C}$ ), while PG showing peaks at $267^{\circ} \mathrm{C}$ (Cakić et al., 2006, 2007 c). All DTG curves showed that there are different stages of degradation which are not perceptible in TG curves, showing the close relation and mutual influence between degradation of hard and soft segments.

The degradation profiles of PU cast films obtained from water-based dispersions were influenced by the type of chain extender, length of the hard segment and type of catalysts. The presence of more selective catalysts, which formed urethane linkages with higher hard segment proportions, had a marked influence on the degradation of the polymers, especially in elevated quantities, improving the thermal stability of the materials. The DTG curves showed that the length of the hard segment had a strong influence on the thermal profile of the samples as a whole. The type of chain extender, forming urethane linkages, affected the whole process of degradation and the presence of more selective catalyst improved the thermal resistance of the chains.

\section{Water-based PUD based on glycolized products obtained from recycled poly (ethylene terephthalate)}

Recycling of polymers has received a great deal of attention (Atta et al., 2006, 2007). Although several methods have been proposed for recycling waste poly(ethylene terephthalate) (PET), it is suggested that the most attractive method is glycolysis of chemicals into the corresponding monomers or raw chemicals that could be reused for the production of plastics or other advanced meterials (Patel et al., 2007).

Two-stage PUD synthesis was applied: the first, glycolysis of PET using different types of glycols (PG), triethylene glycol(TEG) and poly(ethylene glycol) (PEG 400), with different molar ratio of PET repeating unit to glycol (1:2 and 1:10); the second, preparation of PUD of the products formed.

PUD are prepared by anionic dispersion process (Cakić et al., 2011), using IPDI, glycolyzed products, DMPA as potential ionic center which allow water dispersibility and ethylene dimine (EDA) as chain extender.

\subsection{Experimental}

Example 1 of glycolysis reaction: Small pieces of PET waste $(100 \mathrm{~g})$, equivalent to $0.5 \mathrm{~mol}$ repeating unit (mol.wt. $192 \mathrm{gmol}^{-1}$ ) were added to $88.64 \mathrm{~g} \mathrm{PG}$ (mol.wt. 76.09 gmol-1 $\left.^{-1}\right), 173.07 \mathrm{~g}$ TEG (mol.wt.150 gmol$^{-1}$ ) or 461.5 g PEG 400 (mol.wt. 400 gmol$^{-1}$ ), such that the molar ratio of PET repeating unit to glycol was 1:2. 
Example 2 of glycolysis reaction: In the second experimental runs of depolymerisation, appropriate amount of PET waste were added to $396.1 \mathrm{~g}$ PG, $750 \mathrm{~g}$ TEG or $2173 \mathrm{~g}$ PEG 400, so that molar ratio of PET repeating unit to glycol was 1:10. These mixtures (with different molar ratio PET/glycol) and $0.5 \mathrm{wt} \%$ zinc acetate (based on the weight of PET as transesterification catalyst) were charged to a glass reactor, which was fitted with stirrer, reflux condenser, nitrogen inlet and temperature controller. This reactor was immersed in an oil bath and the content of the reaction kettle was heated at $190^{\circ} \mathrm{C}$ for $2 \mathrm{~h}$, subsequently the temperature was raised to $210{ }^{\circ} \mathrm{C}$ until all the solids disappeared.

The obtained glycolyzed oligoester polyols were analysed by the hydroxyl value (HV) determination according to the conventional acetic anhydride/pyridine method (Cakić et al., 2011). The hydroxyl value of the oligoester polyol obtained in the glycolysis reaction based on molar ratio of PET repeating unit to glycol, 1:2, with PG was HBPG $=490 \mathrm{mg} \mathrm{KOH} / \mathrm{g}$, TEG $\mathrm{HBTEG}_{\mathrm{TE}}=370 \mathrm{mg} \mathrm{KOH} / \mathrm{g}$ and PEG HBPEG400 $=297 \mathrm{mg} \mathrm{KOH} / \mathrm{g}$.

The hydroxyl value of the oligoester polyol obtained in the glycolysis reaction based on molar ratio of PET repeating unit to glycol, 1:10, with PG was $\mathrm{HBPG}=201 \mathrm{mg} \mathrm{KOH} / \mathrm{g}$, TEG HBTEG=209 mg KOH/g and PEG HBPeg400=192 mg KOH/g.

\subsection{Synthesis of PUD based on glycolyzed products with molar ratio PET/glycol, $1: 2$}

Anionic PUD based on glycolyzed products with molar ratio PET/glycol, 1:2, were prepared by modified acetone process. Acetone was added to the prepolymer and the dispersion is formed by the addition of water to this solution. Procedure for synthesis of anionic PUD has been developed adjusting the molar ratio of DMPA as a hydrophilic monomer to IPDI as 1:3.3. Mass of oligoester polyol, obtained by PET glycolysis, according to example 1, with a hydroxyl number which is equivalent to the hydroxyl number of $0.06 \mathrm{~mol}$ of poly(propylene glycol) PPG1000 (110 mg KOH/g), was for PG 15 g, TEG 20 g, poly (ethylene glycol) (PEG 400) 25 g.

The oligoester polyol and hydrophilic monomer (8 g, equ. $0.06 \mathrm{~mol})$ was mixed in the cosolvent DMF (50:50 w/w), in a 250-ml round four-neck glass reactor connected to a stirrer, a thermometer, a reflux condenser and a nitrogen gas inlet. The reaction was carried out at $70{ }^{\circ} \mathrm{C}$ for $30 \mathrm{~min}$ to obtain a homogeneous mixture and the uniform distribution of hydrophilic monomer to PU backbone. IPDI (44.4 g, equ. $0.2 \mathrm{~mol})$ and catalyst DBTDL $(0.03 \%$ of the total solid) were added to the homogenized mixture at $80{ }^{\circ} \mathrm{C}$ for about $4 \mathrm{~h}$ until the amount of residual NCO groups reached a theoretical value, as determined by the dibutyl amine backtitration method. To reduce the viscosity and obtain a homogenous mixture of NCO prepolymers, acetone was added $50 \mathrm{wt} \%$ to the solid reaction mass. The theoretical value of NCO groups for PUD based on oligoester polyol obtained from the glycolysis with molar ratio of PET repeating unit to glycol, 1:2, was $19.2 \%$ for PG, $17.7 \%$, for TEG and $16.1 \%$ for PEG.

After obtaining completely NCO terminated prepolymer, the mixture was cooled to $60{ }^{\circ} \mathrm{C}$ and the carboxylic groups in hydrophilic monomer were neutralized with TEA (DMPA equ). TEA was dissolved in NMP by stirring the solution for $60 \mathrm{~min}$. 
PU anionomer was cooled to $30{ }^{\circ} \mathrm{C}$ then dispersed in water (50\% of total mass) under high speed stirring for $30 \mathrm{~min}$. The rate of water addition to the mixture was carefully controlled, to obtain a stable inversion. Upon completing the phase inversion, EDA $(0.03 \mathrm{~mol})$ was added for $60 \mathrm{~min}$. at $35{ }^{\circ} \mathrm{C}$. PUD of about $30 \mathrm{wt} \%$ solids was obtained upon removal of acetone by rotary vacuum evaporation at $35^{\circ} \mathrm{C}$.

\subsection{Synthesis of PUD based on glycolyzed products with molar ratio PET/glycol,} $1: 10$

Anionic PUD based on glycolyzed products with molar ratio PET/glycol, 1:10, were prepared by prepolymer mixing method in two steps: synthesis of NCO-terminated prepolymers and the preparation of dispersions by introducing anionic centers to aid dispersions (Athawale \& Kulkarni, 2009). The prepolymer mixing method has been developed adjusting the molar ratio of DMPA to IPDI as 1:3, which was increased compared to the previous procedure. Mass of oligoester polyol, obtained by PET glycolysis, according to example 2, with a hydroxyl number which is equivalent to the hydroxyl number of 0.1 mol of poly(propylene glycol) PPG1000 (110 mg KOH/g), was for PG $54.5 \mathrm{~g}$, TEG 52.6 g, poly (ethylene glycol) (PEG 400) $57.3 \mathrm{~g}$. The mass of oligoester polyols and hydrophilic monomer was the same as in the previous procedure, but the mass of IPDI was (66.6 g, equ.0.3 mol). determined by molar ratio of a hydrophilic monomer to IPDI as 1:3. In order to obtain NCO terminated prepolymer, synthesis was controlled by determing the NCO groups by the dibutyl amine back-titration method until a theoretical value was achieved. The theoretical value of NCO groups for PUD based on oligoester polyol obtained from glycolysis with molar ratio of PET repeating unit to glycol, 1:10, was $14.2 \%$ for PG, $13.7 \%$, for TEG and $13.9 \%$ for poly(ethylene glycol). The neutralization of the carboxylic groups in hydrophilic monomer, extension chain with EDA and the preparation of stable dispersion was done as in the previous modified acetone process. The solid content in this dispersion was $30 \%$.

Films were prepared by casting the aqueous dispersions on the glass surface and allowing them to dry at room temperature for 7 days and then at $60{ }^{\circ} \mathrm{C}$, for $12 \mathrm{~h}$ (Coutinho, 1996, 2003). Films were cast by $100 \mu \mathrm{m}$ applicators from the solutions onto glass surface $(7 \mathrm{~cm} \times 2$ $\mathrm{cm})$ to obtain dry film thickness of 25-30 $\mu \mathrm{m}$, making demoulding impossible. After demoulding, the films were kept into a desicator to avoid moisture. TG experiments were performed in a Perkin-Elmer TG-7 analyser. Film samples about $20 \mathrm{mg}$ were placed in a platinum sample pan and heated from 30 to $600{ }^{\circ} \mathrm{C}$, with an air flow of $200 \mathrm{~mL} \mathrm{~min}^{-1}$ and heating rates of $10{ }^{\circ} \mathrm{C} \mathrm{min}^{-1}$. During the heating period, the weight loss and temperature difference were recorded as a function of temperature.

TGA was used to analyze decomposition behavior of cured films of PUD synthesized with glycolized products obtained from PET waste. TG curves are depicted in Figs. $4 \mathrm{a}, 4 \mathrm{c}$ and $4 \mathrm{e}$ (curve marked as 1 a show lower molar ratio of PET / glycol (1:2) in the glycolized oligoester) represents the degradation of PUD influenced by the variation of oligoester polyols. It was verified that the thermal stability was influenced by glycol type and different molar ratio of PET repeating unit to glycol in glycolysis reaction. Figs. $4 \mathrm{~b}, 4 \mathrm{~d}$ and $4 \mathrm{f}$ depict the behavior of corresponding differential weight loss (DTG) curves. 


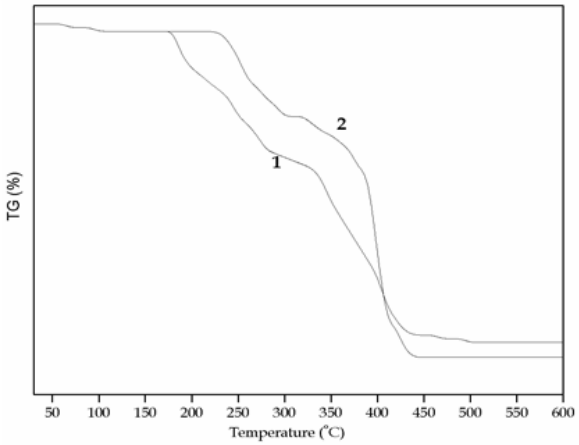

(a)

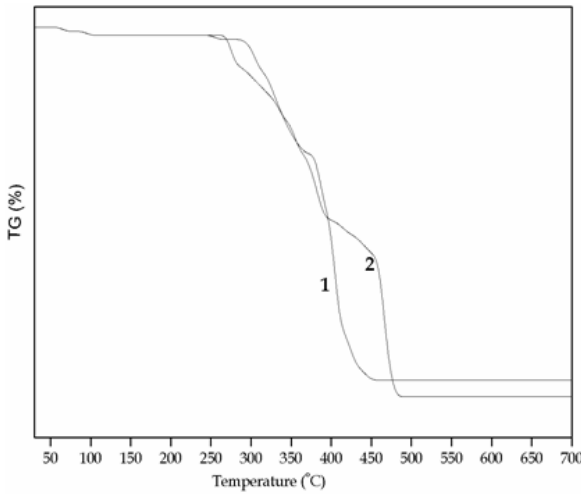

(c)

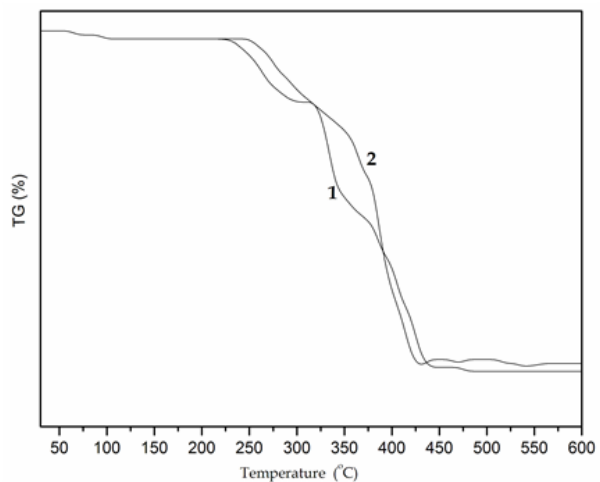

(e)

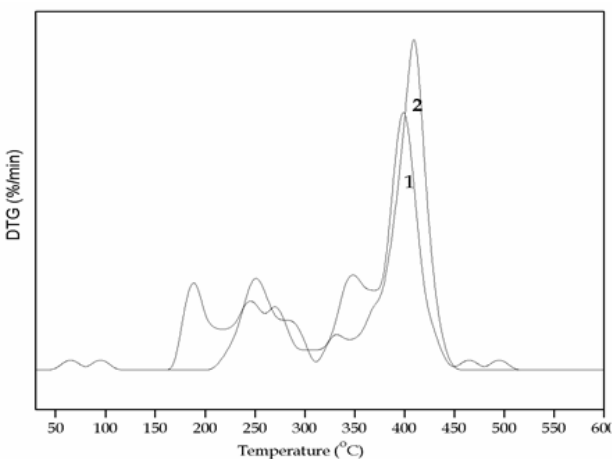

(b)

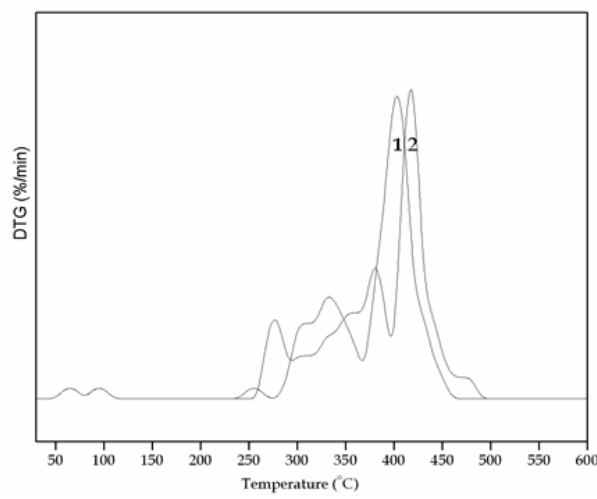

(d)

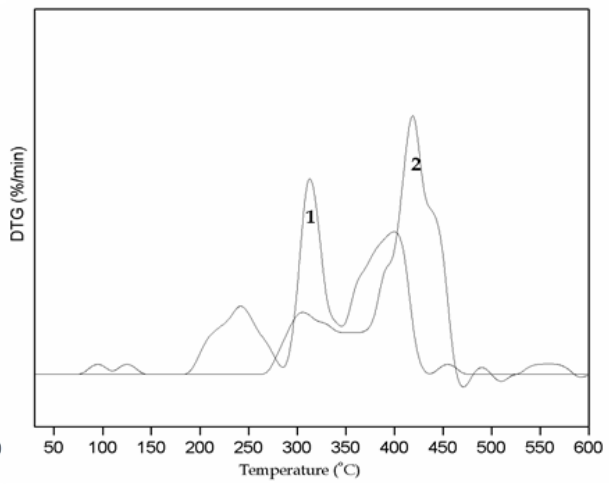

(f)

Figure 4. TG curves (a) and DTG curves (b) of PUD synthesized from glycolized oligoester PET/PG with molar ratio 1:2 (1) and 1:10 (2). TG curves (c) and DTG curves (d) of PUD synthesized from glycolized oligoester PET/TEG with molar ratio 1:2 (1) and 1:10 (2). TG curves (e) and DTG curves (f) of PUD synthesized from glycolized oligoester PET/poly(ethylene) glycol 400 with molar ratio 1:2 (1) and 1:10 (2). 
PUD (synthesized from glycolized oligoester PET/PG (1:2), Fig. 4b) synthesized from depolymerised oligoesters with lower molar ratio of PET repeating unit to glycol in glycolysis reaction showed lower thermal stability in the initial stage of degradation may be due to the presence of greater amount of aromaticity in polyester backbone which makes the PU chains susceptible to scission and relieves the structure crowing (Athawale \& Kulkarni, 2010). In later stage (above $300{ }^{\circ} \mathrm{C}$ ), it showed enhanced thermal stability. It has also been proved that two or three peaks of first decomposition were well correlated with the higher value of polydispersity of GPC results (1.65), for oligoester polyols PET/PG (1:2) compared to the values of polydispersity (1.20), for oligoester polyols PET/PG(1:10). Curve marked as 2 , in Figures $4 \mathrm{~b}$, $4 \mathrm{~d}$ and $4 \mathrm{f}$, which shifted third decomposition step temperature to the higher values, shows that glycolized oligoester obtained with higher molar ratio of PET/glycol of 1:10 have better thermal stability of obtained PUD.

Because of the presence of oligoester polyols wich lower molecular weight in glycolysis reaction and a diamine were used in the synthesis of PUD, two kinds of hard segments are formed, urethane and urea. It has been estabilished that the urethanes have lower thermal resistance than urea and therefore the first decomposition process at about $190-250{ }^{\circ} \mathrm{C}$ and the second at about $270-290{ }^{\circ} \mathrm{C}$ of PUD should correspond to the urethane and urea hard segments, respectively. The decomposition temperature of the soft segment is observed at $400-430^{\circ} \mathrm{C}$. The decomposition temperature for investigated samples are listed in Table 1.

\begin{tabular}{|lc|c|c|c|c|}
\hline \multicolumn{2}{|c|}{ Sample } & \multicolumn{2}{|c|}{ First decomposition } & $\begin{array}{c}\text { Second } \\
\text { decomposition }\end{array}$ & $\begin{array}{c}\text { Third } \\
\text { decomposition }\end{array}$ \\
\cline { 3 - 7 } & & $T_{10 n}\left({ }^{\circ} \mathrm{C}\right)$ & $T_{1 \max }\left({ }^{\circ} \mathrm{C}\right)$ & $T_{2}\left({ }^{\circ} \mathrm{C}\right)$ & $T_{3}\left({ }^{\circ} \mathrm{C}\right)$ \\
\hline PET/PG & $(1: 2)$ & $\begin{array}{l}190.2 \\
247.0\end{array}$ & 270.7 & 349.7 & 400.3 \\
\hline PET/TEG & $(1: 2)$ & - & 288.0 & 335.1 & 401.9 \\
\hline PET/PEG400 & $(1: 2)$ & 248.6 & - & 316.5 & 403.4 \\
\hline PET/PG & $(1: 10)$ & 251.7 & 288.1 & 327.0 & 411.3 \\
\hline PET/TEG & $(1: 10)$ & - & 277.6 & 379.6 & 416.4 \\
\hline PET/PEG400 & $(1: 10)$ & - & - & 305.4 & 422.4 \\
\hline
\end{tabular}

Table 1. Temperature of decomposition of PUD

The degradation profile of PUD was dependent on mole ratios of PET to glycol in glycolyzed products.

The samples based on PET/glycol, at molar ratio of 1:10, had better thermal stability than samples based on PET/glycol, at molar ratio of 1:2. The higher values of temperature for third decomposition stage, for samples with molar ratio of 1:10, probably is due to the increased length of glycol in glycolyzed oligoester polyol. 


\section{Waterborne PUD based on polycarbonate diols (PCD)}

The polyols used in PUD synthesis are of polyether-, polyester-, polycaprolactone- and polycarbonate- origin. The use of individual types of polyol chains and their functionalities depend on the purpose of the potential use, e.g.; PUD made from polyesters can have slightly elevated strength and oil resistance compared to polyether-based PUD and have been largely used in PU paints as they exhibit outstanding resistance to light and aging. Polyether polyols are susceptible to light and oxygen when hot, however, they improve water dispersion, and impart chain flexibility (Gunduz \& Kisakurek, 2004). The use of PCD in PUD, as compared to other polyols, imparts better hydrolysis resistance, improved ageing and oil resistance, excellent elastomeric properties even at low temperature, improved mechanical properties, good weathering and fungi resistance (Garcia et al., 2010). PCD used as the soft segment component in PUD synthesis are usually obtained from dimethylcarbonate or ethylene carbonate and a linear aliphatic diol (Foy et al., 2009). The properties of PUD are related to their chemical structure (Cakić et al., 2009; Athawale \& Kulkarni, 2010) and are mainly determined by the interactions between the hard and soft segments, and the interactions between the ionic groups (Garcia et al., 2011). The properties of PUD are strongly influenced by composition and ionic content, an important target in an investigation of the role of the composition (Lee at al., 2004, 2006).

\subsection{Experimental}

Water-based PUD derived from IPDI, with different molar ratio PCD to DMPA, were prepared by the modified dispersing process. The ionic group content in PU-ionomer structure was varied by changing the amount of the internal emulsifier, DMPA (4.5, 7.5 and $10 \mathrm{wt} \%$ to the prepolymer weight).

Three waterborne PUD were prepared using $\mathrm{NCO} / \mathrm{OH}=1.5$ by method in which the dispersing procedure was modified (Lee et al., 2006). In the modified procedure only the dispersing stage was varied compared to the standard procedure. The prepolymer solution was mixed with a small amount of deionized water for dispersion of polymer in water. Solvent was added for reducing the viscosity, if necessary.

Into a $250 \mathrm{ml}$ glass reaction kettle, equipped with a mechanical stirrer containing a torque meter, a thermometer, a condenser for reflux and nitrogen gas inlet, was added $60 \mathrm{~g}(0.03$ $\mathrm{mol}$ ) of PCD (dried under vacuum at $120^{\circ} \mathrm{C}$ ); and 4,8 or $12 \mathrm{~g}(0.03,0.06$ or $0.09 \mathrm{~mol}$ ) of DMPA dispersed in $30 \mathrm{ml}$ DMF. The reaction mixture was heated at $70{ }^{\circ} \mathrm{C}$ for $0.5 \mathrm{~h}$ to obtain a homogeneous mixture. This step is important for the resulting equal uniform distribution of hydrophilic monomer, DMPA, on PU backbone. After that 20, 32 or $40 \mathrm{~g}(0.09,0.15$ or 0.18 $\mathrm{mol}$ ) of IPDI and DBTDL (0.03 wt. \% of the total solid) were added to the homogenized mixture and stirred at $80{ }^{\circ} \mathrm{C}$ for $2.5 \mathrm{~h}$. Dibutyl amine back titration method was used for the determination of the reaction time necessary to obtain completely NCO-terminated prepolymer. Then the mixture was cooled down to $60{ }^{\circ} \mathrm{C}$ and carboxylic groups (DMPA equiv) were neutralized with TEA dissolved in NMP (2 wt \% of the total reaction mass) by stirring the reaction mixture at $60^{\circ} \mathrm{C}$ for $1 \mathrm{~h}$. 
Subsequently, the prepolymer solution was mixed with $0.3 \mathrm{ml}$ of deionised water for dispersion step-by-step. Stirring was increased during the addition of water, and the mixture was diluted with NMP. Waterborne PUD was obtained by drop-wise addition of water to the mixture in order to obtain PUD with solid content of about $30 \%$ at $30{ }^{\circ} \mathrm{C}$ for $1 \mathrm{~h}$. The chain extension was carried out with solution of 0.9 or $1.8 \mathrm{~g}(0.015$ or $0.03 \mathrm{~mol})$ of EDA in $2 \mathrm{ml}$ of deionised water at $35^{\circ} \mathrm{C}$ for $1 \mathrm{~h}$. The mixture was heated to $80^{\circ} \mathrm{C}$ under vacuum in order to remove NMP and to obtain PUD with solid content of about $30 \%$.

The thermal stability of PU was determined by TG. The DTG thermogram of cured films of PUD based on PCD showed several degradation steps (Fig.5b). Detailed analysis of the thermogram is represented in Table 2. The decrease in the DMPA content produces a slight increase in the decomposition temperature (Fig.5a). However, the degradation mechanism was very complex due to the different stability of the hard and soft segments.

The removal of residual water due to incomplete drying of PU was produced at around $130{ }^{\circ} \mathrm{C}$. The DTA thermogram of the used aliphatic PCD shows the main degradation at $350{ }^{\circ} \mathrm{C}$ and other less important at $265^{\circ} \mathrm{C}$ (Garcia, 2010, 2011). Because this diol and EDA were used in the synthesis of PU, two kinds of groups in hard segments have been formed, i.e., urethane and urea ones. The decomposition temperature of PUD is mostly influenced by the chemical structure of the component having the lowest bond energy (Coutinho et al., 2003; Cakic et al.,2009). The urethane bond has lower thermal resistance than the urea bond and thus the first decomposition process at about $280{ }^{\circ} \mathrm{C}$ corresponds to the beginning of the urethane part of hard segment degradation and second at about $300{ }^{\circ} \mathrm{C}$ to the degradation of the urea part of hard segment. The degradation in PU at $264-268{ }^{\circ} \mathrm{C}$ is characteristic of the polyol. The degradation of soft segment (mainly composed of polyol) is produced at $329-338^{\circ} \mathrm{C}$. The soft and hard segments content were quantified from the weight loss at above mentioned temperatures $\left(265{ }^{\circ} \mathrm{C}\right.$ from PCD degradation, $280{ }^{\circ} \mathrm{C}$ and $300{ }^{\circ} \mathrm{C}$ correspond to the urethane and urea hard segment degradation, and $330^{\circ} \mathrm{C}$ from degradation of soft segment).

According to Table 2, the decrease in DMPA content produced a slight increase in the decomposition temperature and a decrease in the weight loss for the decomposition of urethane and urea hard segments, which can be ascribed to a decrease in the amount of hard segment. The decomposition temperature of the soft segments is produced at $329-338{ }^{\circ} \mathrm{C}$ and the weight loss increase by decreasing DMPA content 20.9 and 23.5 to $6.2 \mathrm{wt} . \%$ in PU ionomers.

\begin{tabular}{|c|c|c|c|c|c|c|}
\hline \multirow{2}{*}{$\begin{array}{c}\text { Sample } \\
\text { PUD }\end{array}$} & \multicolumn{2}{|c|}{ Residual water } & \multicolumn{2}{c|}{ Soft segment } & \multicolumn{2}{c|}{ Hard segment } \\
\cline { 2 - 7 } & $\mathrm{T}\left({ }^{\circ} \mathrm{C}\right)$ & $\begin{array}{c}\text { Weight loss } \\
(\mathrm{wt} \%)\end{array}$ & $\mathrm{T}\left({ }^{\circ} \mathrm{C}\right)$ & $\begin{array}{c}\text { Weight loss } \\
(\mathrm{wt} \%)\end{array}$ & $\mathrm{T}\left({ }^{\circ} \mathrm{C}\right)$ & $\begin{array}{c}\text { Weight } \\
\text { loss }(\mathrm{wt} \%)\end{array}$ \\
\hline $4.5 \% \mathrm{DMPA}$ & 129.8 & 1.1 & $268 ; 329$ & $10.1 ; 20.9$ & $282 ; 301$ & $23.5 ; 45.4$ \\
\hline $7.5 \% \mathrm{DMPA}$ & 137.3 & 1.0 & $264 ; 338$ & $3.8 ; 23.5$ & $290 ; 304$ & $17.5 ; 53.7$ \\
\hline $10 \%$ DMPA & 128.8 & 0.9 & $267 ; 331$ & $10.5 ; 6.2$ & $280 ; 301$ & $22.9 ; 59.3$ \\
\hline
\end{tabular}

Table 2. Temperature of decomposition and weight loss of PUD (obtained by TG measurements) 
The decrease in DMPA content produces a decrease in the hard segment content of PU ionomers. The resistance to thermal degradation of PU ionomer increased by decreasing the DMPA content due to the lower hard segment content.

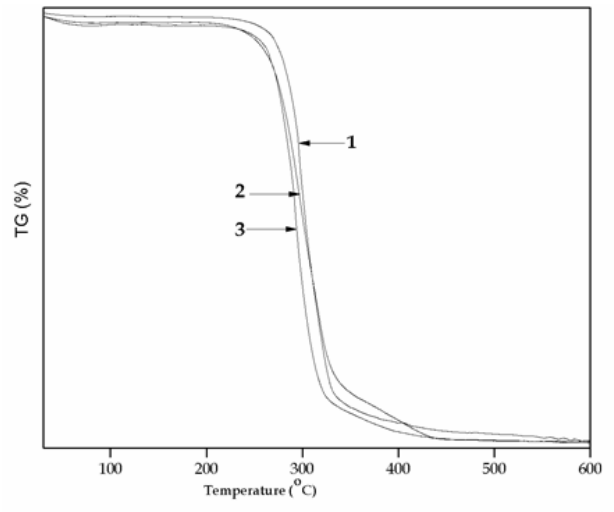

(a)

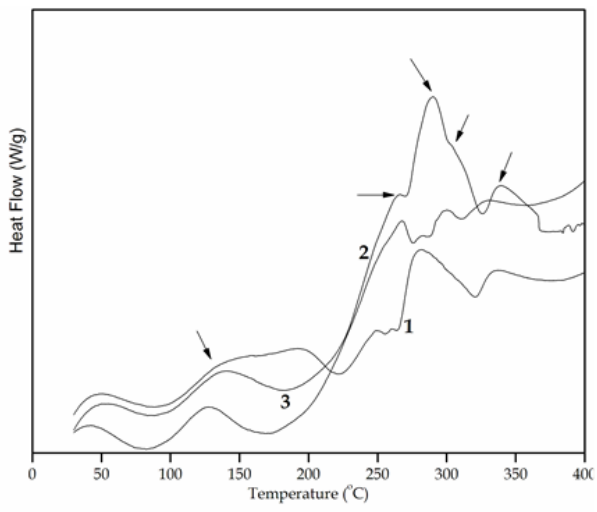

(b)

Figure 5. TGA curves (a) and DTA curves (b) of cured films of PUD based on PCD with 4.5\% DMPA (1), 7.5\% DMPA (2), 10\% DMPA (3).

\section{Conclusions}

The wide application of PUD makes necessary better understanding of the chemistry-structure relationship that improves the thermal stability as this is important prerequisite to obtain tailor-made products for high performance applications. In this work, the investigation on thermal degradation of PUD with well-defined architectures indicated that diol types and DMPA content had great influence on thermal stability. PUD with lower DMPA content has shown enhanced thermal stability. The degradation profiles of PU aqueous dispersions were influenced by the type of chain extender, length of the hard segment and type of catalysts. The TG curves showed that the length of the hard segment had a strong influence on the thermal profile of the samples as a whole. The possibility for using glycolysis products of waste PET in PUD manufacturing was confirmed. The effects of glycol type and the different mole ratios of PET to glycol on thermal properties of PUD have been described. The degradation profile of the dispersions was dependent on mole ratios of PET to glycol in glycolyzed products. The samples based on (PET/glycol molar ratio 1:10) have shown enhanced thermal properties, which can be ascribed to increased length of glycol in glycolyzed oligoester polyol.

\section{Author details}

Suzana M. Cakić and Olivera Z. Ristić

University of Niš, Faculty of Technology, Leskovac, Serbia

Ivan S. Ristić

University of Novi Sad, Faculty of Technology, Novi Sad, Serbia 


\section{References}

[1] Atta A.M.; Abdel-Raouf M.E.; Elsaeed S.M.; Abdel-Azim A.A. (2006) Curable resins based on recycled poly(ethylene terephthalate) for coating applications. Prog. Org. Coat., 55, pp. 50-59.

[2] Atta A.M.; El-Kafrawy A.F.; Aly M.H.; Abdel-Azim A.A. (2007) New epoxy resins based on recycled poly(ethylene terephthalate) as organic coatings. Prog. Org. Coat., 58, pp. 13-22.

[3] Athawale V.D. \& Kulkarni M.A. (2009) Preparation and properties of urethane/acrylate composite by emulsion polymerization technique. Prog. Org. Coat., 65, pp. 392-400.

[4] Athawale V.D. \& Kulkarni M.A. (2010) Polyester polyols for waterborne polyurethanes and hybrid dispersions. Prog. Org. Coat. 67, pp. 44-54.

[5] Bechara I. (1998) Formulating with polyurethane dispersions. Eur. Coat. J., 4, pp. 236243.

[6] Blank W.J.; He Z.A. \& Hessell E.T. (1999) Catalysis of the isocyanate-hydroxyl reaction by non-tin catalysts. Prog. Org. Coat., 35, pp. 19-29.

[7] Blank W.J. \& Tramontano V.J. (1996) Properties of crosslinked polyurethane dispersions. Prog. Org. Coat., 27(1), pp. 1-15.

[8] Cakić S. M.; Lačnjevac Č.; Stamenković J.; Ristić N.; Takić Lj.; Barać M.; Gligorić M. (2007 a) Effects of the acrylic polyol structure and the selectivity of the employed catalyst on the performance of two-component aqueous polyurethane coatings. Sensors, 7(3), pp. 308-318.

[9] Cakić S.M.; Nikolić G.S.; Stamenković J.V. (2007 b) Thermo-oxidative stability of waterborne polyurethanes with catalysts of different selectivity evaluated by nonisothermal thermogravimetry. J. Serb. Chem. Soc., 72(7), pp. 723-735.

[10] Cakić S.M.; Nikolić G.S.; Lačnjevac Č.; Gligorić M.; Rajković M. (2007 c) The thermal degradation of aqueous polyurethane with catalysts of different selectivity. Prog. Org. Coat., 60(2), pp. 112-116.

[11] Cakic S.; Lačnjevac Č.; Rajković M.B.; Rašković Lj.; Stamenković J. (2006 a) Reticulation of Aqueous Polyurethane Systems Controlled by DSC Method. Sensors, Vol. 6, No. 5, pp. 536-545.

[12] Cakic S.; Lačnjevac Č.; Nikolić G.; Stamenković J.; Rajković M.B.; Gligorić M.; Barać M. (2006 b) Spectroscopic Characteristics of Highly Selective Manganese Catalysts in Acqueous Polyurethane Systems. Sensors, 6, pp. 1708-1720.

[13] Cakić S.; Ristić I.; Djordjević D.; Stamenković J.; Stojiljković D. (2010) Effect of the chain extender and selective catalyst on thermooxidative stability of aqueous polyurethane dispersions. Prog. Org. Coat., 67, pp. 274-280.

[14] Cakić S.; Ristić I.; M-Cincović M.; Nikolić N.; Ilić O.; Stojiljković D.; B-Simendić J. (2011) Glycolyzed products from PET waste and their application in synthesis of polyurethane dispersions. Prog. Org. Coat., In press, doi: 10.1016/j. porgcoat.2011.11.024.

[15] Cakić S.; Stamenković J.; Djordjević D.; Ristić I. (2009) Synthesis and degradation profile of cast films of PPG-DMPA-IPDI aqueous polyurethane dispersions based on selective catalysts. Polym. Degrad. Stab., 94, pp. 2015-2022. 
[16] Chang T.C.; Chiu Y.S.; Chen H.B.; Ho S.Y. (1995) Degradation of phosphorus-containing polyurethanes. Polym. Degrad. Stab., 47, pp. 375-381.

[17] Chattopadhyay D.K. \& Webster D.C. (2009) Thermal stability and flame retardancy of polyurethanes. Prog. Polym. Sci. 34, pp. 1068-1133.

[18] Collong W.; Gobel A.; Kleuser B.; Lenhard W.; Sonntag M. (2002) 2K waterborne clearcoat-a competition between crosslinking and side reactions. Prog. Org. Coat., 45, pp. 205-209.

[19] Coutinho F.M.B. \& Delpech M.C. (1996) Some properties of films cast from polyurethane aqueous dispersions of polyether-based anionomer extended with hydrazine. Polym. Test.,15, pp. 103-113.

[20] Coutinho F.M.B.; Delpech M.C.; Alves T.L.; Ferreira A.A. (2003) Degradation profiles of cast films of polyurethane and poly(urethane-urea) aqueous dispersions based on hydroxyterminated polybutadiene and different diisocyanates. Polym. Degrad. Stab., 81, pp. 19-27.

[21] Delpech M.C. \& Coutinho F.M.B. (2000) Waterborne anionic polyurethanes and poly(urethane-urea)s: influence of the chain extender on mechanical and adhesive properties. Polym. Test., 19, pp. 939-952.

[22] Dieterich D. (1981) Aqueous Emulsions, Dispersions and Solutions of Polyurethanes; Synthesis and. Properties. Prog. Org. Coat., 9, pp. 281-340.

[23] Dulog L. \& Storck G. (1966) Die oxydation von polyepoxiden mit molekularem sauerstoff, Macomol. Chem., 91, pp. 50-73.

[24] Fambri L.; Pegoretti A.; Gavazza C.; Penati A. (2000) Thermooxidative Stability of Different Polyurethanes Evaluated by Isothermal and Dynamic Methods. J. Appl. Polym. Sci., 81, pp. 1216-1225.

[25] Foy E.; Farrell J.B.; Higginbotham C.L. (2009) Synthesis of Liner Aliphatic Polycarbonate Macroglycols Using Dimethylcarbonate. J. Appl. Polym. Sci., 111, pp. 217-227.

[26] Garcia-Pacios V.; Costa V.; Colera M.; J. Martin-Martinez M. (2010) Affect of polydispersity on the properties of waterborne polyurethane dispersions based on polycarbonate polyol. Int. J. Adhes. Adhes., 30, pp. 456-465.

[27] Garcia-Pacios V.; Costa V.; Colera M.; Martin-Martinez J.M. (2011) Waterborne polyurethane dispersions obtained with polycarbonate of hexanediol intended for use as coatings. Prog. Org. Coat., 71, pp. 136-146.

[28] George Woods, (1987). The ICI Polyurethanes Book, $2^{\text {nd }}$ Edition, Wiley, New York.

[29] Gunduz G. \& Kisakurek R.R. (2004) Structure-Property study of waterborne polyurethane dispersions with Different hydrophilic content and polyols. J. Disper. Sci.Technol., 25 (2), pp. 217-228.

[30] Hepburn C. (1992) Polyurethane Elastomers, Second ed., Elsevier, New York.

[31] Jacobs, P.B. \& Yu, P.C. (1993) Two-Component Waterborne Polyurethane Coatings. J.Coat. Tech., 65 (822), pp. 45-50.

[32] Jang J. Y.; Jhon Y.K.; Cheong I.W.; Kim J.H. (2002) Effect of process variables on molecular weight and mechanical properties of water-based polyurethane dispersion. Colloids Surf. A- Physicochem. Eng. Aspects, 196, pp. 135-143. 
[33] Kim B.K. \& Min L.Y. (1994) Aqueous dispersion of polyurethanes containing ionic and nonionic hydrophilic segments. J. Appl. Polym. Sci., 54, pp. 1809-1815.

[34] Kim B. K. (1996) Aqueous polyurethane dispersions. Coll. Polym. Sci., 274, pp. 559-611

[35] Lee D.K.; Tsai H.B.; Tsai R.S. (2006) Effect of Composition on Agueous polyurethane Dispersions Derived from polycarbonatediols. J. Appl. Polym. Sci., 102, pp. 4419-4424.

[36] Lee D.K.; Tsai H.B.; Wang H.H.; Tsai R.S. (2004) Aqueous Polyurethane Dispersions Derived from Polycarbonatediols. J. Appl. Polym. Sci., 94, pp. 1723-1729.

[37] Lee H.T.; Hwang Y.T.; Chang N.S.; Huang C.C.T.; Li H.C. (1995) Waterborne, HighSolids and Powder Coatings Symposium, New Orleans, pp. 224.

[38] Lu M.G.; Lee J.Y.; Shim M.J.; Kim S.W. (2002) Thermal Degradation of Film Cast from Aqueous Polyurethane Dispersions. J. Appl. Polym. Sci., 85, pp. 2552-2558.

[39] Oertel G. (1985) Polyurethane Handbook, Hanser Publishers, Munich.

[40] Patel M.R.; Patel J.V.; Mishra D.; Sinha V.K. (2007) Synthesis and Characterization of Low Volatile Content Polyurethane Dispersion from Depolymerised Polyethylene Terphthalate. J. Polym. Eviron., 15, pp. 97-105.

[41] Pielichowski K.; Slotwinska D.; Dziwinski E. (2004) Segmented MDI/HMDI based polyurethanes with lowered flammability. J. Appl. Polym. Sci., 91, pp. 3214-3224.

[42] Prime R.B.; Burns J.M.; Karmin M.L.; Moy C.H.; Tu H.B. (1988) Applications of DMA and TGA to quality and process control in the manufacture of magnetic coatings. $J$. Coat. Technol., 60, pp. 55-60.

[43] Ramesh S. \& Radhakrishna G. (1994) Synthesis and characterization of polyurethane ionomers. Polym. Sci., 1, pp. 418-423

[44] Randell D. \& Lee S. (2000) Polyurethane Book, $2^{\text {nd }}$ Editors, John Wiley \& Sons, New York.

[45] Rothause J. W. \& Nechtkam K. (1987) Advances in Urethane Science and Technology, 10, pp. 121

[46] Rosthauser J.W. \& Nachtkamp K.J. (1986) Waterborne polyurethanes. J.Coat. Fabrics., 16, pp. 39-79.

[47] Scaiano J. C. (1989) Laser Photolysis in Polymer Chemistry. Degradation and Stabilization of Polymers. Elsevier, Amsterdam.

[48] Stamenković J.; Cakić S.; Nikolić G. (2005) Study of the catalytic selectivity of an aqueous two-component polyurethane system by FTIR spectroscopy. Chem. Ind., 57, pp. 559-562.

[49] Tawa T. \& Ito S. (2006) The Role of Hard Segments of Aqueous Polyurethane-urea Dispersion in Determining the Colloidal Characteristics and Physical Properties. Polym. J., 38(7), pp. 686-693.

[50] Wang T.L. \& Hsieh T.H. (1997) Effect of polyol structure and molecular weight on the thermal stability of segmented poly(urethaneureas). Polym. Degrad. Stab., 55, pp. 95-102. 